\title{
La gestion des émotions face aux situations stressantes à l'école : les finissants stagiaires en éducation préscolaire et enseignement primaire se sentent-ils prêts? \\ Managing emotions while coping with stressful situations at school: Are graduating preschool and elementary school teachers ready? \\ La gestión de las emociones ante situaciones estresantes en la escuela: ¿los pasantes finalistas en educación preescolar y enseñanza primaria se siente listos?
}

\section{Marie-Andrée Pelletier}

Volume 43, numéro 2, automne 2015

Le stress à l'école

URI : https://id.erudit.org/iderudit/1034492ar

DOI : https://doi.org/10.7202/1034492ar

\section{Aller au sommaire du numéro}

\section{Éditeur(s)}

Association canadienne d'éducation de langue française

\section{ISSN}

1916-8659 (numérique)

Découvrir la revue

\section{Citer cet article}

Pelletier, M.-A. (2015). La gestion des émotions face aux situations stressantes à l'école : les finissants stagiaires en éducation préscolaire et enseignement primaire se sentent-ils prêts? Éducation et francophonie, 43(2), 201-218.

https://doi.org/10.7202/1034492ar

\section{Résumé de l'article}

Être en mesure de faire face à toutes les violences (physique, morale, verbale, etc.) pour ne pas perdre le contrôle de certaines situations en contexte scolaire demande une formation psychologique très particulière qui n'était pas nécessaire autrefois (Voirol, 1998). Plusieurs enseignants débutants affirment qu'ils ont de la difficulté en début de carrière à répondre aux exigences de la pratique; d'autres ont des attentes irréalistes quant à l'enseignement et aux élèves (Martineau et Presseau, 2007). Dans ces conditions, il importe de se pencher sur la place qu'occupe la formation psychologique dans les programmes de formation initiale des futurs enseignants. Cet article présente une partie des résultats issus d'une étude sur les besoins de formation psychologique perçus par onze stagiaires finissantes de quatrième année au baccalauréat en éducation préscolaire et enseignement primaire (BEPEP), plus spécifiquement les résultats liés à la gestion des émotions dans la profession. Les résultats montrent que ces stagiaires souhaitent, dans leur formation, apprendre à maîtriser le stress devant les difficultés de la profession enseignante, à connaître la gamme d'émotions pouvant être vécues avec les différentes personnes impliquées dans le milieu scolaire, à décrocher à la maison et à prendre du recul devant ces difficultés, mais également à réaliser des analyses réflexives plus poussées au regard de la gestion des émotions en enseignement.
Tous droits réservés (C) Association canadienne d'éducation de langue française, 2015
Ce document est protége par la loi sur le droit d'auteur. L'utilisation des services d’Érudit (y compris la reproduction) est assujettie à sa politique d'utilisation que vous pouvez consulter en ligne. 


\title{
La gestion des émotions face aux situations stressantes à l'école: les finissants stagiaires en éducation préscolaire et enseignement primaire se sentent-ils prêts?
}

\author{
Marie-Andrée PELLETIER \\ Université du Québec à Rimouski, campus de Lévis, Québec, Canada
}

\section{RÉSUMÉ}

Être en mesure de faire face à toutes les violences (physique, morale, verbale, etc.) pour ne pas perdre le contrôle de certaines situations en contexte scolaire demande une formation psychologique très particulière qui n'était pas nécessaire autrefois (Voirol, 1998). Plusieurs enseignants débutants affirment qu'ils ont de la difficulté en début de carrière à répondre aux exigences de la pratique; d'autres ont des attentes irréalistes quant à l'enseignement et aux élèves (Martineau et Presseau, 2007). Dans ces conditions, il importe de se pencher sur la place qu'occupe la formation psychologique dans les programmes de formation initiale des futurs enseignants. Cet article présente une partie des résultats issus d'une étude sur les besoins de formation psychologique perçus par onze stagiaires finissantes de quatrième année au baccalauréat en éducation préscolaire et enseignement primaire (BEPEP), plus spécifiquement les résultats liés à la gestion des émotions dans la profession. Les résultats montrent que ces stagiaires souhaitent, dans leur formation, apprendre 
à maîtriser le stress devant les difficultés de la profession enseignante, à connaître la gamme d'émotions pouvant être vécues avec les différentes personnes impliquées dans le milieu scolaire, à décrocher à la maison et à prendre du recul devant ces difficultés, mais également à réaliser des analyses réflexives plus poussées au regard de la gestion des émotions en enseignement.

\section{ABSTRACT}

\section{Managing emotions while coping with stressful situations at school: are graduating preschool and elementary school teachers ready?}

Marie-Andrée PELLETIER

University of Quebec in Rimouski, Lévis campus, Quebec, Canada

Being able to deal with any type of violence (physical, moral, verbal, etc.) to avoid losing control of certain situations in the school context, requires very specific psychological training that was not previously required. (Voirol, 1998). Many new teachers say that that they have trouble early in their careers meeting the requirements of the practice, while others have unrealistic expectations about education and students (Martineau and Presseau, 2007). In these circumstances, it is important to consider the role of psychological education in initial training programs for future teachers. This article presents some results of a study on the needs for psychological training perceived by 11 trainees graduating from the $4^{\text {th }}$ year of a Bachelor of Education in preschool and primary education (BEPPE), more specifically results related to managing emotions in the profession. The results show that they wish their training could teach them how to master the stress of dealing with the difficulties involved in the teaching profession, to learn about the range of emotions that can be experienced by the various people involved in the school environment, to let go of work when they get home and distance themselves from these problems, and also to be able to make more elaborate reflexive analyses on managing the emotions related to teaching. 


\section{RESUMEN}

\section{La gestión de las emociones ante situaciones estresantes en la escuela: ¿los pasantes finalistas en educación preescolar y enseñanza primaria se siente listos?}

Marie-Andrée PELLETIER

Universidad de Quebec en Rimouski, campus de Levis, Quebec, Canadá

Ser capaz de confrontar todas las violencias (física, moral, verbal, etc.) y no perder el control en ciertas situaciones en contexto escolar, exige una formación sicológica muy particular que antes no se consideraba como necesaria (Voirol 1998). Varios maestros debutantes afirman que al comenzar su carrera les pareció difícil responder a las exigencias del oficio, otros presentan expectaciones irrealistas con respecto a la enseñanza y a los alumnos (Martineau y Presseau, 2007). En tales condiciones, parece ser importante interrogarse sobre el lugar que ocupa la formación sicológica en los programas de formación magisterial. Este artículo presenta una parte de los resultados provenientes de un estudio sobre las necesidades en formación sicológica percibidas por 11 pasantes que terminan su cuarto año de bachillerato en educación preescolar y enseñanza primaria (BEPEP), más específicamente, se muestran los resultados relacionados con la gestión de las emociones en la profesión. Los resultados demuestran que los pasantes desean que su formación pueda enseñarles a controlar las emociones que pueden ser vividas con las diferentes personas implicadas en el medio escolar, a olvidar los problemas una vez en casa y a distanciarse de dichas dificultades, pero también a realizar análisis reflexivos más elaborados con respecto a la gestión de las emociones durante el ejercicio de su profesión.

\section{Introduction}

Janot-Bergugnat et Rascle (2008) évoquent que le stress est l'une des dimensions importantes du métier d'enseignant dont les programmes de formation doivent tenir compte. Selon Royer, Loiselle, Dussault, Cossette et Deaudelin (2001), le stress est un état négatif et se distingue de la motivation à relever les défis. Il s'avère très nuisible dans la profession et il peut entraver la productivité des enseignants. Cette réalité a été soulignée par onze stagiaires finissantes de quatrième année ayant terminé le stage IV au baccalauréat en éducation préscolaire et enseignement primaire (BEPEP). Le but était de connaître leur perception de ce qui se fait actuellement dans les programmes de formation au regard de leur formation psychologique. Ces différents points de vue ont permis de mettre en lumière des aspects fort pertinents pour 
l'avancement des connaissances dans le domaine de l'éducation, plus précisément à l'égard de la gestion des émotions en enseignement.

Dans cet article, la problématique concernant certaines lacunes en formation initiale sera brièvement exposée. Ensuite, la formation psychologique sera abordée selon trois concepts clés: motivation, représentations et émotions. Une partie de la méthodologie de recherche d'où proviennent les données sera décrite et les résultats et les éléments de synthèse de la discussion de ces derniers seront présentés. Les besoins de formation psychologique seront alors proposés pour permettre aux formateurs, aux enseignants et aux décideurs des programmes en formation à l'enseignement de réfléchir à leur pratique et d'offrir un encadrement réflexif (Altet, 2002). Ces résultats sont un apport important pour outiller les formateurs, mais ils ouvrent également la voie à d'autres réflexions quant à la place de la gestion des émotions en formation initiale au regard des situations stressantes pouvant être vécues dans la profession enseignante. Dès la formation initiale, il apparaît important de s'attarder à la compréhension de ses réactions et actions souvent amorcées par les émotions (Goyette, 2009). La gestion des émotions sera facilitée dans des situations problématiques et le taux de stress amoindri puisque l'enseignant devient capable de relativiser les choses et de faire des choix judicieux (Goleman, 1999).

\section{Problématique}

Les débuts dans l'enseignement peuvent être caractérisés par le stress de se retrouver seul devant un groupe d'élèves et d'assumer toutes les responsabilités liées à la tâche (Pelletier, 2013). Royer et al. (2001) soulignent, entre autres, la charge de travail, le manque de temps, le comportement inadéquat des élèves et les relations pauvres ou difficiles avec les autres membres du personnel, la direction ou les parents. Gendron (2011) souligne d'ailleurs que les facteurs liés à la tâche, à l'organisation du travail, aux relations de travail, à l'environnement physique et technique et à l'environnement socioéconomique exposent les enseignants à un stress.

Ces situations ont des incidences sur leur travail, notamment en ce qui concerne leur type de stress, leur satisfaction à l'égard du métier, mais surtout leur persévérance dans la profession (Leroux, 2009). Selon Delsemme (2004), il existe encore beaucoup trop de lacunes dans la formation, de sorte que les enseignants débutants se sentent démunis pour affronter une profession aussi complexe. Ce qui est vécu réellement diffère parfois de ce qui est attendu, et cet écart, plus il devient grand, soulève des difficultés quant à l'acceptation et à l'adaptation à la situation. En effet, selon Dumoulin (2013), plusieurs enseignants débutants vivent un choc de la réalité qui entraîne d'importantes remises en question de leurs idéaux éducatifs et de leur choix professionnel. Les attentes ou les idéaux personnels associés à l'enseignement sont souvent en rupture avec la réalité du métier et des écoles, et cela peut être à l'origine de frustrations et de remises en question pouvant conduire à l'abandon de la profession (Mukamurera, 2011). Un répondant de l'étude de Sauvé (2012) sur l'attrition des enseignants au Québec, qui a abandonné la profession enseignante, souligne 
qu'«on est amené à gérer des humains, mais on apprend à gérer des humains avec des livres. Il y a peut-être un manque d'application, de pratique quand on suit nos cours à l'université» (p. 117).

Depuis plusieurs années, des recherches sont menées sur ce qu'on appelle le travail émotionnel à risque pour la santé. Selon Dantzer (1988), le terme "émotion" désigne globalement les sentiments que chacun peut reconnaître en lui-même par introspection ou prêter aux autres par extrapolation. Les résultats de recherches effectuées par l'équipe de Paul Ekman (1972) établissaient que l'être humain, peu importe sa culture, possède six émotions universelles: la joie (ou le plaisir), la peur, la colère, la surprise, le dégoût et la tristesse. Selon Janot-Bergugnat et Rascle (2008), à l'heure où le tabou sur le stress des enseignants commence à être levé, c'est une des dimensions importantes de leur métier dont il faudrait à l'avenir tenir compte dans les programmes de formation. Ils ajoutent que devant un élève difficile l'enseignant peut se mettre en colère, avoir peur, se sentir las, vivre toute une palette d'émotions négatives qui vont venir interférer dans sa prise de décision pour résoudre un problème. On demande de plus en plus aux enseignants d'accepter de nouvelles responsabilités, de gérer des élèves avec des caractéristiques particulières (difficultés d'apprentissage, d'adaptation ou un handicap). Esteve (1999) précise que «non seulement on leur demande d'enseigner, mais aussi de veiller à l'équilibre psychologique et affectif de leurs élèves» (p. 130). À cet égard, il est donc pertinent d'interroger des stagiaires finissants sur les besoins de formation psychologique liés à la gestion des émotions pour concevoir des stratégies adaptatives centrées sur les émotions et en réponse aux différentes situations stressantes pouvant être vécues à l'école.

\section{Cadre conceptuel}

\section{Concept de formation psychologique en enseignement}

Avant d'entamer une étude sur les besoins de formation psychologique des stagiaires terminant le BEPEP, il apparaît nécessaire de cibler les informations importantes à considérer pour situer le concept de formation psychologique en enseignement, et ce, selon les différentes définitions qui lui sont associées. En guise de résumé, voici quelques éléments essentiels à retenir. D'une part, plusieurs auteurs insistent sur le fait que l'essence de la formation psychologique repose sur l'établissement d'une relation pédagogique permettant à l'enseignant de maitriser efficacement ses émotions (Calin, 2011; Latry, 2004; Nimier, 1996). D'autre part, la formation psychologique fait évoluer les représentations (Baillauquès, 1990; Latry, 2004; Nimier, 1996) des enseignants sur leur métier, sur leurs élèves, sur ce qu'est la transmission des connaissances et les prépare à l'évolution du monde actuel. De plus, la motivation (Calin, 2011; Latry, 2004) fait appel à des compétences qui reposent sur la connaissance de soi ainsi que sur la perception de la valeur des activités pédagogiques en formation des enseignants. Selon Calin (2011), le travail de mise en adéquation de l'art d'enseigner et de la complexité du métier relève alors du champ de la formation psychologique (FP). 
La littérature théorique et empirique reflète peu de consensus en ce qui a trait à la conceptualisation et à la définition du concept de FP en enseignement (Baillauquès, 1990; Blanchard-Laville et Toux-Alavoine, 2001; Calin, 2011; Fellay, 1992; Lamarre, 2004; Latry, 2004; Nimier, 1996). À la suite de la présentation des différentes définitions abordées par les auteurs, on constate que la FP peut être explorée sous différents angles. En effet, le manque de consensus empirique amène à examiner la formation psychologique selon des phénomènes différents. Cependant, la majorité des chercheurs s'entendent généralement pour dire que la FP en enseignement est nécessaire pour remédier aux situations déstabilisantes de la profession (Baillauquès, 1990; Calin, 2011; Latry, 2004; Lamarre, 2004; Nimier, 1996). Il ne s'agit pas simplement de gérer un groupe d'élèves, mais d'apprendre auparavant à se gérer soi-même devant le groupe, ce que propose une FP en enseignement. Trois concepts semblent particulièrement liés à la formation psychologique: la motivation, les représentations et les émotions.

\section{Motivation}

Dès leur formation, les futurs enseignants doivent clarifier leurs motivations afin d'entretenir des attentes réalistes par rapport à la profession (Mukamurera, 2011). La formation universitaire a un effet important sur la motivation d'enseigner, puisqu'elle est le lieu ultime de socialisation professionnelle et de construction identitaire (Mukamurera, 2011). Selon Deci et Ryan (1985), il existe deux types de motivation, soit la motivation intrinsèque et la motivation extrinsèque. La première aide l'individu à s'autodéterminer et à s'autoréguler pour faire face aux difficultés d'une situation. La seconde naît d'éléments de l'environnement qui influencent ses décisions et ses actions. Goleman (1999), cité par Goyette (2009, p. 26), souligne quatre composantes de la motivation: l'exigence de la perfection (l'effort investi pour atteindre ses objectifs), l'engagement (savoir partager les objectifs du groupe), l'initiative (être prêt à saisir les opportunités) et l'optimisme (poursuivre ses objectifs malgré les obstacles et les déconvenues).

La définition qui s'applique dans le cadre de cette étude est celle qui considère la motivation comme «l'ensemble de forces internes et externes qui déclenchent et déterminent la forme, la direction, l'intensité et la durée (persistance) des comportements liés au travail» (Pinder, 2008, traduction libre, p. 11). De plus en plus de chercheurs (Latham et Pinder 2005; Mitchell et Daniels, 2003) portent attention aux phénomènes émotionnels pour définir la motivation, sachant que la variation des émotions a un impact sur le comportement d'un individu au travail. À titre d'exemple, si ce dernier vit une situation de stress, la motivation peut moduler la réponse à cet événement tel qu'il est accueilli, soit avec résignation, détresse, colère ou encore avec des sentiments de défi ou de plaisir. Selon Selye et Turcotte (1976), le stress engendré par les conflits, la haine et la frustration serait évité et la joie serait atteinte si les individus accordaient plus d'attention à la compréhension des bases naturelles de la motivation et de la conduite. 


\section{Représentations du métier}

Kagan (1992) utilise l'expression «connaissances personnelles» pour définir les représentations. Selon cette auteure, les connaissances personnelles peuvent influencer les futurs enseignants à deux moments précis, soit au cours de leur formation universitaire ou lors de leur insertion professionnelle. Toujours d'après Kagan (1992), les représentations peuvent prendre la forme d'images, de perceptions, d'impressions, mais aussi d'opinions préconçues et de préjugés. Selon Raynal et Rieunier (1997), il y a lieu de distinguer représentation et connaissance, la première étant plus circonstancielle, contextualisée et ayant des fins spécifiques, la seconde étant plus permanente et moins dépendante du contexte, d'une fonction ou d'une tâche. La représentation est donc une construction intellectuelle, individuelle et collective, qui permet de donner du sens à une situation (Saint-Jacques, Chené, Lessard et Riopel, 2002). Par ailleurs, on voit ici une distinction intéressante et nécessaire à faire entre "perception» et "représentation», car ces termes sont parfois confondus. La perception renvoie à une réalité présente dans l'action, tandis que la représentation renvoie à une réalité absente et reconstruite à partir des caractéristiques à la fois individuelles, sociales et du milieu (Depeau, 2006). Toutefois, il n'y a pas de perception sans représentation.

La rencontre d'événements non familiers et les écarts entre la réalité du milieu et celle qu'ils imaginaient constituent également des composantes de l'expérience qui peuvent les placer dans une situation de déséquilibre affectif et cognitif (Lamarre, 2004). Selon Goyette (2009), les émotions font partie du quotidien des enseignants et influencent leurs perceptions du milieu, des élèves, des pairs, ainsi que leurs représentations personnelles du métier et leur sentiment de compétence. Une représentation alarmiste des conditions de stress est susceptible de limiter les enseignants dans les stratégies efficaces pour les gérer (Doudin, Curchod-Ruedi et Moreau, 2011). La gestion des émotions a une incidence directe sur les décisions que les enseignants prennent en lien avec une situation et, selon Goleman (1999), pour qu'un enseignant ou une enseignante puisse s'épanouir dans sa profession, il est primordial qu'il soit capable de gérer ses émotions afin qu'elles ne lui nuisent pas dans sa pratique.

\section{Gestion des émotions}

La formation psychologique liée à la gestion des émotions suppose un travail sur la prise de conscience et la réflexion pour comprendre de nouvelles situations (analyse de pratiques), développer ses capacités d'analyse réflexive, prévoir les situations professionnelles susceptibles d'être déplaisantes et améliorer ses capacités d'adaptation en s'inscrivant dans une perspective de développement personnel et professionnel (Martin, Morcillo et Blin, 2004), et ce, dès la formation initiale des futurs enseignants. Or, la gestion des émotions fait référence à la capacité de l'enseignant de tenir compte des sentiments éprouvés par les personnes impliquées dans le métier (enseignants, élèves, parents, direction, etc.) en tentant de les orienter dans un sens qui favorise la bonne gestion d'une situation et de modifier ses propres émotions afin de les rendre compatibles avec le travail à réaliser. Selon Salovey et Mayer 
(1990), il s'agit, notamment, de reconnaître les émotions des autres, mais aussi ses propres émotions, en étant capable de les analyser, de les maîtriser et de les mettre au service d'un but.

Selon Nimier (1996), il est important qu'une formation psychologique sur la gestion des émotions permette aux futurs enseignants de reconnaître leurs émotions et d'accepter de les gérer. Garder le contrôle des émotions perturbatrices permet à un individu de rester calme, positif, concentré et imperturbable devant des situations éprouvantes afin de prendre une décision éclairée malgré le stress (Goyette, 2009). Westen (2000) estime que "si une personne ne peut pas directement modifier la situation stressante, elle peut alors essayer de changer la perception qu'elle en a ou bien les émotions que cette situation provoque» (p. 619). Lazarus et Folkman (1984) distinguent quant à eux des stratégies d'ajustement (coping) qui peuvent correspondre aux efforts orientés vers la gestion des émotions, soit la prise de distance et la minimisation des menaces, la réévaluation positive, l'auto-accusation, la fuite-évitement ou, encore, la maîtrise de soi.

\section{Question de recherche et objectif}

$\mathrm{Au}$ regard de la question générale «Quels sont les besoins de formation psychologique chez les finissants en éducation préscolaire et enseignement primaire?", l'objectif général est de cibler, de décrire et d'analyser les besoins de formation psychologique chez les finissants au BEPEP. Ce texte, qui est un abrégé d'une thèse de doctorat en éducation (Pelletier, 2013), présente les résultats liés à la gestion des émotions face aux situations stressantes à l'école.

\section{Méthodologie}

Cette étude se situe dans le courant qualitatif. Elle est interprétative, car elle interprète le phénomène selon le sens que les participantes, finissantes en éducation préscolaire et enseignement primaire, confèrent à leur propre formation psychologique. Elle est exploratoire, car elle aspire à connaître et à découvrir ce que pensent les finissantes, futures enseignantes, dans le but de générer des pistes d'intervention à explorer.

\section{Échantillonnage}

Léchantillonnage privilégié dans cette étude est dit intentionnel (Deslauriers, 1991). En effet, l'échantillon de cas typiques choisis fournit des renseignements à partir de quelques cas représentatifs de l'ensemble. L'approche a ainsi été faite auprès des groupes de stagiaires terminant le BEPEP de la cohorte 2008-2012 de l'Université du Québec à Rimouski (UQAR). Dans le cadre de cette recherche, un échantillon de onze stagiaires finissantes dont la moyenne d'âge était de 24 ans et ayant complété le stage IV au BEPEP a été constitué selon une participation volontaire. 


\section{Protocole de recherche}

Pour favoriser la mise en place d'une relation propice à l'échange, des questions simples posées par la chercheure invitent d'abord le participant à parler de façon libre et ouverte, ce qui favorise un climat de confiance et le partage de réflexions. Les entretiens sont d'une durée d'environ une heure et sont enregistrés à l'aide d'un magnétophone. Le lieu n'est pas une préoccupation de cette recherche, mais il convient de prévoir un endroit calme, loin de certaines distractions. La plupart des entrevues se sont donc déroulées à l'université même où étudie la chercheure.

Afin de conserver la profondeur et la rigueur du paradigme de l'approche qualitative, l'entretien semi-dirigé est choisi comme principal instrument de collecte de données de la recherche, sachant qu'il permet d'aborder, entre autres, une liste de catégories préétablies, de formuler des questions à partir de ces dernières et de les appliquer selon un ordre choisi. Au regard de la gestion des émotions en enseignement, des questions ouvertes sont posées aux participantes. À titre d'exemple, la participante doit répondre à un type de question tel que:

"Quelles compétences auriez-vous souhaité développer pour apprendre à

gérer vos émotions et de quelles façons auriez-vous pu les développer?»

Dans le cadre de cette étude, ces questions sont formulées d'après les catégories créées à partir des résultats obtenus lors d'une première collecte de données recueillies auprès des enseignantes débutantes selon une technique du groupe nominal.

\section{Analyse des données}

$\mathrm{Au}$ regard de cette étude sur les besoins de formation psychologique chez les finissants au BEPEP, l'analyse des données s'inspire du modèle de Miles et Huberman (2003) tout en tenant compte des éléments importants du modèle de Deslauriers (1991), soit la première étape de la lecture et relecture du matériel, la seconde étape de la déconstruction des données ainsi que la troisième étape de la reconstruction et de la synthèse. La première étape exige du chercheur qu'il s'approprie le matériel et connaisse ainsi les données recueillies. La seconde étape consiste à découper et à réduire les informations en unités de sens comparables, donc à tracer une première esquisse des thèmes significatifs. Les données d'entrevues, réduites à un format unique à la suite du processus de codification, deviennent alors plus faciles à interpréter. À la troisième étape, une synthèse générale est formulée et conduit à la distinction entre les catégories ainsi que les éléments qui les forment. Une signification est ainsi dégagée du phénomène étudié. Afin de respecter la confidentialité, un code est attribué à chacune des participantes. D'autre part, au regard du modèle cyclique de Miles et Huberman (2003), le format narratif est utilisé pour la présentation des données qualitatives, mais des extraits d'entretien sont également présentés pour illustrer l'analyse des données brutes utilisées.

Il convient de préciser que des exigences de la rigueur méthodologique associée à cette approche ont été tenues pour compte, soit des critères de fiabilité, crédibilité, transférabilité et constante interne (Gohier, 2004). Pour assurer la fiabilité, on a d'abord fait appel aux enseignants débutants en insertion professionnelle selon la 
technique du groupe nominal (TGN) afin de déterminer les compétences psychologiques à maîtriser pour faire face à la complexité du métier. Ensuite, ces compétences sont examinées chez les finissantes au BEPEP à l'aide des entretiens semi-structurés. Ces deux instruments s'avèrent complémentaires du fait qu'ils permettent une compréhension du phénomène étudié. Le croisement des données analysées et des éléments du cadre théorique et conceptuel vise alors à assurer la crédibilité des résultats au moment de l'interprétation des résultats. Les résultats peuvent permettre une transférabilité dans certains contextes. Toutefois, il est difficile de prétendre que les résultats de la recherche sont généralisés à la population, sachant que l'échantillon se restreint à onze finissantes. De plus, l'échantillon se limite à une université. Les résultats ne peuvent représenter l'ensemble des finissants sur ce territoire, bien qu'ils puissent assurer une certaine représentativité. La constante interne est assurée par le choix des différents modes de collecte et d'analyse et a fait l'objet d'une vérification externe auprès d'experts dont le comité de direction associé à cette thèse, mais également les professeurs attitrés à la scolarité du doctorat. Au préalable, la méthode d'analyse des données a été clarifiée et l'opérationnalisation de chacune d'elles précisée. Bien que ce choix méthodologique s'explique par le fait d'approfondir et de mettre en perspective les besoins de formation psychologique des finissants, il n'en reste pas moins qu'il demeure dans un contexte de subjectivité.

\section{Présentation des résultats}

À l'égard des résultats obtenus à la suite de l'analyse et de l'interprétation, il se dégage de la synthèse générale quatre éléments qui retiennent l'attention: 1) la mâ̂trise du stress devant les difficultés vécues dans la profession enseignante, 2) l'identification des émotions pouvant être vécues avec les différentes personnes impliquées dans le milieu scolaire, 3) la manière de décrocher à la maison et de prendre du recul devant les difficultés vécues dans la profession et 4) la réalisation d'analyses réflexives dans le cadre de la formation pratique au regard des émotions vécues.

\section{Maîtrise du stress devant les difficultés vécues}

À l'égard de la gestion des émotions, la maîtrise du stress devant les difficultés vécues dans la profession enseignante est le premier besoin mentionné par plus de $80 \%$ des stagiaires finissantes. Elle concerne le désir de disposer de stratégies et de conseils pour maîtriser son stress devant les difficultés vécues.

... dans la formation des maîtres, c'est d'être formés, d'être prêts psychologiquement à entrer [...] sur le marché du travail. Donc, être prêts à affronter justement la précarité [...] parfois c'est les émotions qu'on vit avec les enfants, avec les parents. (FP1-04)

Dans le même ordre d'idées, un répondant précise que la formation doit préparer aux réalités de la profession en apprenant aux futurs enseignants et enseignantes à faire face aux imprévus : 
On peut être compétent professionnellement, mais sans avoir le mental pour. Donc, gérer le stress, la violence [...] ça peut être comment réagir aux imprévus. Toutes nos réactions, c'est d'être formé à réagir adéquatement. (FP1-09)

À l'unanimité, les participantes interrogées ont d'ailleurs souligné l'exemple de la phase de suppléance qui provoque parfois, chez le nouvel enseignant ou la nouvelle enseignante, du stress ou même encore un état d'anxiété.

... J'aurais aimé développer des cours qui auraient pu permettre de gérer cette anxiété-là. En suppléance, j'aurais aimé apprendre à gérer nos émotions, quand un élève te rit dans 'face [sic] ou t'insulte. [...] La formation aurait pu aider dans le sens d'apprendre à calmer le jeu [...]. C'est peut-être d'être outillé face à ça. (GE11-08)

\section{Émotions vécues avec les différentes personnes impliquées}

Le second besoin est l'identification des émotions pouvant être vécues avec les différentes personnes impliquées dans le milieu scolaire. Actuellement, les stagiaires finissantes disent savoir comment intervenir techniquement auprès des élèves en difficulté et en crise, mais près de $80 \%$ souhaitent connaître plus largement les émotions pouvant être vécues dans ces contextes et apprendre à les gérer en relation avec d'autres partenaires, soit les parents, les enseignants, la secrétaire, les collègues ou encore la direction.

Bien peut-être avec les parents [...] on l'a effleuré, mais dans nos stages aussi. J'étais tout le temps accompagné de mon enseignante associée. Je n'ai jamais intervenu [sic] avec un parent qui était en colère, parce que la note de son enfant n'est pas bonne. J'aurais peut-être préféré davantage avoir plus de soutien là-dessus en formation. (CI21-04)

... On nous apprend à travailler avec les élèves, [...] mais y a plein d'autres personnes autour de nous qui faut [sic] apprendre à travailler avec, en tant que suppléante, stagiaire. (CI21-08)

Dans un autre ordre d'idées, des stagiaires finissantes proposent d'ailleurs que les formateurs provoquent des situations qui leur permettent de sortir de leur zone de confort lors des stages ou encore de les exposer à des exemples de situations dans lesquelles les enseignants vivent certains types d'émotions avec les différents partenaires.

\section{Manière de décrocher à la maison et de se distancier}

Le troisième besoin, la manière de décrocher à la maison et de se distancer devant les difficultés vécues dans la profession, correspond au fait de savoir comment décrocher à la maison et se distancier devant les difficultés vécues par les élèves ou les familles.

... Moi je suis du genre à beaucoup amener les problèmes chez moi [...]. J'vous dirais qu'en suppléance les enfants nous testent, on ne se fera pas de 
cachette, donc peut-être de nous dire justement, bon... les enfants vont vous tester, faites-vous-en pas, créez-vous un mur. [...] quand les enfants y me cherchent [sic] c'est sûr qu'émotionnellement je ne sais pas comment je vais réagir. (GE11-03)

Près de $50 \%$ des participantes souhaitent apprendre à « décrocher» de leur journée une fois à la maison, mais surtout à se distancier des réalités de la profession. En effet, elles savent que leur frustration ne doit pas paraître devant un événement qui survient en gestion de classe ou encore lorsqu'ils sont confrontés à des cas familiaux particuliers; mais elles souhaitent aussi appliquer des stratégies pour ne pas transposer les problèmes à la maison. Certaines participantes mentionnent qu'ils souhaitent être outillés pour éviter l'épuisement, le burnout et même une certaine culpabilité lorsqu'ils ne réussissent pas à avoir une belle journée de suppléance, par exemple.

$\mathrm{O} k$, mais comment on fait pour s'immuniser contre ça, là on le dit pas. [...] Puis même nous dire: regardez, la fin de semaine décrochez... Comment avoir une carrière saine, parce que comment ça arrive le burnout, ça arrive comme ça, comment l'éviter? Regardez, faites ça, donnez-vous à mettons [sic] tant d'heures par soir pour mettre sur votre travail [sic], la fin de semaine décrochez... (GE11-02)

\section{Réalisation d'analyses réflexives au regard des émotions vécues}

Le quatrième besoin, la réalisation d'analyses réflexives dans le cadre de la formation pratique au regard des émotions vécues, implique que les stagiaires finissantes puissent réaliser des analyses réflexives au regard des émotions vécues et qu'elles sachent comment agir.

J'pense que l'autoévaluation c'est extrêmement formateur puisque de se dire je commence mon baccalauréat, puis quand y m'arrive [sic] telle chose, ça affecte comment j'enseigne. [...] puis j'pense de se dire moi j'ai de la misère côté stabilité émotionnelle, si je rapporte les cas à la maison. Bien j’pense que si t'en es conscient au début de ta formation, tu vas pouvoir travailler au cours de tes stages. Puis tu ne feras peut-être pas l'erreur quand tu vas être rendue en suppléance. (GE11-06)

Il semble qu'actuellement la formation permette aux futurs enseignants de réfléchir sur eux-mêmes en apprenant à gérer les crises auprès des élèves, en prenant du recul ou même en faisant un retour sur leurs propres compétences professionnelles à la fin de leur baccalauréat (Pelletier, 2013). Cependant, près de $50 \%$ des participantes souhaitent que cette pratique réflexive soit davantage centrée sur les émotions.

... prendre le temps d'analyser chaque situation qui arrive dans les stages. Oui, dans certains cours on nous donnait des mises en situation, mais des fois c'est dépassé, c'est comme exagéré. [...] Ce qui est arrivé, c'est qu'on n'a pas pu analyser les moments où qu'on aurait pu, au niveau de la gestion des émotions. Il arrivait une situation, ok, y arrivait ça [sic], j'ai réagi comme ça 
La gestion des émotions face aux situations stressantes à l'école:

les finissants stagiaires en éducation préscolaire et enseignement primaire se sentent-ils prêts?

de telle façon, j'aurais pu réagir de telle façon. Mais pourquoi c'est arrivé comme ça? Pourquoi ce serait mieux? De pousser plus loin dans ça. (GE11-10)

\section{Synthèse de la discussion des résultats}

À l'égard du premier besoin lié à la gestion des émotions en enseignement, la maîtrise du stress devant les difficultés vécues dans la profession enseignante, il semble que les participants souhaitent y être mieux préparés, plus particulièrement dans un contexte de suppléance. Selon Martineau et Presseau (2007), il existe un grand écart entre les conditions d'enseignement dans le contexte d'un stage et celles de la pratique réelle. En effet, lors des stages, l'enseignant associé peut tout simplement intervenir quand le stagiaire éprouve un problème avec le groupe, alors que, lorsque celui-ci entreprendra sa carrière, il devra être autonome. Selon Latry (2004), la formation actuelle ne permet pas d'acquérir une connaissance réelle de son propre fonctionnement psychologique, de repérer ses réactions face à certaines situations, de savoir ce qui fragilise l'enseignant ou l'insécurise, ou ce qui le rassure. Ainsi, la prise de conscience des émotions pouvant être générées dans certaines situations permet de mieux se préparer à les affronter sur le plan émotif. Selon Robert (2007), une émotion est cohérente lorsqu'elle est perçue conforme aux valeurs de l'enseignant et, ainsi, lorsqu'il s'attend à ressentir cette émotion dans la représentation qu'il a de son travail.

Selon les participantes interrogées, l'identification des émotions pouvant être vécues avec les différentes personnes impliquées dans le milieu scolaire ne concerne pas seulement les émotions vécues avec les élèves. Les participantes soulignent, entre autres, qu'elles ne sauraient pas comment intervenir avec la direction ou encore devant un parent en colère. Ils mentionnent que, lors des stages, ils étaient accompagnés de leur enseignant associé. Larivée (2006) souligne d'ailleurs l'insatisfaction des futurs enseignants à l'égard des compétences développées pour intervenir avec les parents, ce qui est, selon lui, le reflet des réalités actuelles où les parents occupent une place plus grande au sein du système d'éducation. Selon St-Vincent (2008), l'enseignant doit s'attendre à un spectre assez large de réactions possibles lorsqu'il communique avec les parents à propos de sujets délicats. En effet, les réactions émotives peuvent s'avérer vives: pleurs, agressivité, blâme sur l'enfant, évitement. Autrement dit, l'enseignant peut se faire surprendre et il doit demeurer vigilant, prévoir les scénarios (St-Vincent, 2008).

Les participantes interrogées mentionnent également la manière de décrocher à la maison et de se distancier devant les difficultés vécues dans la profession comme étant un besoin de formation psychologique. D'après les finissantes interrogées, la formation actuelle n'aborde pas certaines réalités du métier. En effet, des finissantes veulent éviter le «burnout» (GE11-02), la «frustration» (GE12-10), l'«épuisement professionnel» (GE12-11), car ces états s'avèrent néfastes pour leur «santé mentale» (GE12-11). Lafortune, Lafortune et Marion (2011) sont d'avis que "se prémunir de certaines situations de "déplaisir", c'est de savoir s'en distancier» (p. 238), ce qui 
traduit le souhait des stagiaires finissants en ce qui concerne leur capacité à se distancier des situations de classe et à prendre une distance par rapport aux réalités complexes du métier dont celle liée au burnout. Selon Reeve (2012), lorsque les gens reçoivent des rétroactions d'échec ou ont besoin de mobiliser de grands efforts pour faire face aux enjeux de la vie, il leur est d'une grande aide d'avoir des ressources motivationnelles internes pour maintenir un sens de la finalité, mais aussi pour canaliser le stress, calmer le doute ou maintenir des réactions émotionnelles positives. Selon Goyette (2009), il importe que l'enseignant puisse entamer un processus de réflexion de ses pratiques sans être perturbé par des émotions inutiles qu'il n'a pas su mettre de côté pour tirer vraiment un profit de ses introspections.

Travailler la dimension émotionnelle suppose entre autres la prise de conscience et la réflexion pour comprendre de nouvelles situations (analyse de pratiques), pour développer ses capacités d'analyse réflexive, pour prévoir les situations professionnelles susceptibles d'être déplaisantes et pour accroître ses capacités d'adaptation (Martin et al., 2004, p. 601), ce qui traduit les propos du quatrième besoin, la réalisation d'analyses réflexives dans le cadre de la formation pratique au regard des émotions vécues. L’individu devrait effectuer une évaluation personnelle et spécifique de ces états émotionnels. Dans la mesure où l'individu peut réguler ce qu'il pense, il peut influencer sa manière de se sentir et de se comporter (Gaudreau, Royer, Beaumont et Frenette, 2012). Il importe que la formation psychologique puisse leur donner les outils nécessaires à ce travail lié aux émotions, car déjà, lors de la formation, peu d'étudiants prennent le temps de s'arrêter et d'analyser les problèmes qu'ils rencontrent, ce qui amène certains à prendre de mauvaises habitudes comme l'évitement. De plus, les émotions négatives, le stress et la nervosité communiquent de l'information négative à propos des compétences (Bandura, 2003) .

\section{Conclusion}

Cette étude s'interrogeait sur les besoins de formation psychologique ressentis par les stagiaires finissantes au BEPEP en ce qui concerne la gestion des émotions. Actuellement, il semble que la formation permet d'intervenir techniquement auprès des élèves en difficulté ou en crise. Cependant, les participantes interrogées souhaitent que la formation puisse également leur apprendre à gérer leurs émotions auprès des autres partenaires, soit les parents, les enseignants, la secrétaire ou, encore, la direction. De plus, les stagiaires finissantes indiquent qu'elles aspirent à ce que la formation leur fasse connaître les possibilités d'émotions vécues en enseignement. Effectivement, certaines émotions surgissant au travail sont clairement perturbatrices et doivent être contenues. Au regard du métier d'enseignant, les stagiaires évoquent, à plusieurs reprises, le stress lié à la profession.

De surcroît, il y a lieu de s'interroger sur la place de la gestion des émotions dans la formation initiale face aux situations stressantes vécues à l'école, dont celle liée à la phase de suppléance, sachant que cette thématique est citée à plusieurs reprises par les stagiaires finissantes interrogées. Gaudreau et al. (2012) soulignent que «la 
présence d'un fossé important entre les connaissances et la pratique dans le domaine de la discipline et de l'intervention comportementale auprès des élèves rend les enseignants très vulnérables au stress vécu lors de la gestion des comportements difficiles en classe» (p. 89), mais également lorsqu'ils interviennent et éprouvent des difficultés avec les autres collègues.

Dans la mesure où l'ajout d'un cours ne peut être fait, la formation pratique peut, par exemple, intégrer cette thématique dans le cadre des séminaires de stage (Pelletier, 2013). Plusieurs insatisfactions ou frustrations sont ressenties dans le monde de l'enseignement et il importe qu'une formation psychologique puisse offrir l'opportunité aux futurs enseignants et enseignantes de travailler leurs buts personnels servant de modérateur entre les événements négatifs qui surviennent et les émotions. Cet article a permis de connaître les perceptions des finissantes stagiaires quant à leurs besoins de formation psychologique liés à la gestion des émotions en enseignement. Il serait cependant tout aussi pertinent d'étendre ce champ d'investigation aux formateurs et superviseurs universitaires ou aux enseignants associés expérimentés afin de connaître leurs points de vue, ainsi que de souhaiter améliorer les stratégies de gestion des émotions face aux situations stressantes dans le contexte de la profession enseignante.

\section{Références bibliographiques}

ALTET, M. (2002). Quelle(s) professionnalité(s) des formateurs en formation continue? Vers un profil polyidentitaire. Dans M. Altet, L. Paquay et P. Perrenoud (dir.), Formateurs d'enseignants. Quelle professionnalisation? (p. 59-87). Bruxelles: De Boeck.

BAILLAUQUÈS, S. (1990). La formation psychologique des instituteurs. Paris: Presses universitaires de France.

BANDURA, A. (2003). Auto-efficacité: le sentiment d'efficacité personnelle. Paris: De Boeck.

BLANCHARD-LAVILLE, C. et TOUX-ALAVOINE, B. (2001). Le temps de la formation. Dans C. Blanchard-Laville (dir.), Malaise dans la formation des enseignants (p. 167-184). Paris: L’Harmattan.

CALIN, D. (2011). Quelle formation psychologique pour les enseignants? Une approche pragmatique. La nouvelle revue de l'adaptation et de la scolarisation, no $54,109-116$.

DANTZER, R. (1988). Les émotions. Paris: Presses universitaires de France.

DECI, E. L. et RYAN, R. M. (2000). The "What" and "Why" of goal pursuits: Human needs and the self-determination of behavior. Psychological Inquiry, 11(4), 227-268. 
DELSEMME, M. (2004). Évolution des représentations professionnelles de deux étudiantes-stagiaires durant la dernière année de leur stage de formation pratique en français langue seconde, au secondaire (Thèse de doctorat inédite). Université McGill, Montréal.

DEPEAU, S. (2006). De la représentation sociale à la cognition spatiale et environnementale: la notion de "représentation» en psychologie sociale et environnementale. Document inédit, Université Rennes II.

DESLAURIERS, J.-P. (1991). Recherche qualitative. Guide pratique. Montréal: McGraw-Hill.

DOUDIN, P.-A., CURCHOD-RUEDI, D. et MOREAU, J. (2011). Le soutien social comme facteur de protection de l'épuisement professionnel. Dans P.A. Doudin, D. Curchod-Ruedi, L. Lafortune et N. Lafranchise (dir.), La santé psychosociale des enseignants et des enseignantes (p. 11-37). Québec: Presses de l’Université du Québec.

DUMOULIN, M.-J. (2013). Comprendre et voir autrement l'insertion professionnelle. Éducation Canada, 53(1). Récupéré le 17 février 2014 de http://www.cea-ace.ca/education-canada/article/comprendre-et-voir-autrementlinsertion-professionnelle

EKMAN, P. (1972). Universals and cultural differences in facial expressions of emotion. Lincoln: University of Nebraska Press.

ESTEVE, J.-M. (1999). Attitudes des enseignants devant le changement social. Dans H. Caglar (dir.), Etre enseignant: un métier impossible? Hommage à Ada Abraham (p. 123-159). Paris: L'Harmattan.

FELLAY, G. (1992). La formation psychologique des enseignants. Compte rendu d'une enquête faite auprès des instituts de formation en Suisse romande et au Tessin. Éducation et recherche / Bildungsforschung und Bildungspraxis, revue de l’Université de Fribourg, 14(3), 309-325.

GAUDREAU, N., ROYER, É., BEAUMONT, C. et FRENETTE, É. (2012). Gestion positive des situations de classe: un modèle de formation en cours d'emploi pour aider les enseignants du primaire à prévenir les comportements difficiles des élèves. Enfance en difficulté, 1(1), 85-115.

GENDRON, B. (2011). Santé et capital émotionnel du personnel enseignant. Dans P.-A. Doudin, D. Curchod-Ruedi, L. Lafortune et N. Lafranchise (dir.), La santé psychosociale des enseignants et des enseignantes (p. 157-176). Québec: Presses de l'Université du Québec.

GOHIER, C. (2004). De la démarcation entre critères d'ordre scientifique et d'ordre éthique en recherche interprétative. Recherches qualitatives, 24, 3-17.

GOLEMAN, D. (1999). L'intelligence émotionnelle 2. Cultiver ses émotions pour s'épanouir dans son travail. Paris: Robert Laffont. 
GOYETTE, N. (2009). Le plaisir d'enseigner chez des enseignants du secondaire. Quelle place pour les émotions? (Mémoire de maîtrise inédit). Université du Québec à Trois-Rivières.

JANOT-BERGUGNAT, L. et RASCLE, N. (2008). Le stress des enseignants. Paris: Armand Colin.

KAGAN, D. M. (1992). Professional growth among preservice and beginning teachers. Review of Educational Research, 62(2), 129-169.

LAFORTUNE, L., LAFORTUNE, D. et MARION, C. (2011). Le bien-être dans la profession enseignante: une analyse de pratique en équipe de collègues. Dans P.-A. Doudin et al. (dir.), La santé psychosociale des enseignants et des enseignantes (p. 277-250). Québec: Presses de l'Université du Québec.

LAMARRE, A.-M. (2004). Étude de l'expérience de la première année d'enseignement au primaire dans une perspective phénoménologicoherméneutique. Revue des sciences de l'éducation, 24, 19-56.

LARIVÉE, S. J. (2006). Les compétences professionnelles en enseignement: portrait des stages et défis d'une formation en mouvance. Québec français, nº 141, 69-70.

LATHAM, G. P. et PINDER, C. C. (2005). Work motivation theory and research at the dawn of the twenty-first century. Annual Review of Psychology, no 56, 485-516.

LATRY, F. (2004). Les enseignants ont aussi des émotions. Paris: Economica.

LAZARUS, R. S. et FOLKMAN, S. (1984). Stress, appraisal, and coping. New York, NY: Springer.

LEROUX, M. (2009). Étude des relations entre la résilience d'enseignantes et d'enseignants du primaire ouvrant en milieux défavorisés et la réflexion sur la pratique (Thèse de doctorat inédite). Université de Montréal.

MARTIN, F., MORCILLO, A. et BLIN, J.-F. (2004). Le vécu émotionnel des enseignants confrontés à des perturbations scolaires. Revue des sciences de l'éducation, 30(3), 579-604.

MARTINEAU, S. et PRESSEAU, A. (2007, août). Construire et consolider des savoirs et des compétences en début de carrière. Actes du Congrès international d'actualité de la recherche en éducation et en formation, Strasbourg, p. 27-31. Récupéré le 30 décembre 2012 de http://www.congresintaref.org/actes_pdf/ AREF2007_Stephane_MARTINEAU_056.pdf

MILES, M. B. et HUBERMAN, A. M. (2003). Analyse des données qualitatives: recueil de nouvelles méthodes. Bruxelles: De Boeck Université.

MITCHELL, T. R. et DANIELS, D. (2003). Motivation. Dans W. Borman, D. Ilgen et R. Kimoski (dir.). Handbook of psychology (vol. 12, p. 225-254). New York, NY: Wiley. 
MUKAMURERA, J. (2011). Les conditions d'insertion et la persévérance dans la profession enseignante. Dans F. Lacourse, S. Martineau et T. Nault (dir.), Profession enseignante: démarches et soutien à l'insertion professionnelle (p. 37-57). Anjou: CEC.

NIMIER, J. (1996). La formation psychologique des enseignants. Paris : ESF.

PELLETIER, M.-A. (2013). Les besoins de formation psychologique chez les finissants en éducation préscolaire et enseignement primaire (Thèse de doctorat inédite). Université du Québec à Rimouski.

PINDER, C. C. (2008). Work motivation in organizational behavior. New York, NY: Psychology Press.

RAYNAL, F. et RIEUNIER, A. (1997). Pédagogie. Dictionnaire des concepts clés: apprentissage, formation et psychologie cognitive. Paris : ESF.

REEVE, J. (2012). Psychologie de la motivation et des émotions. Bruxelles: De Boeck.

ROBERT, N. (2007). Bien-être au travail. Une approche centrée sur la cohérence du rôle. INRS. Santé et sécurité au travail. Note scientifique et technique no 267.

ROYER, N., LOISELLE, J., DUSSAULT, M., COSSETTE, F. et DEAUDELIN, F. (2001). Le stress des enseignants québécois à diverses étapes de leur carrière. Vie pédagogique, no 119, 5-8.

SAINT-JACQUES, D., CHENÉ, A., LESSARD, C. et RIOPEL, M.-C. (2002). Les représentations que se font les enseignants du primaire de la dimension culturelle du curriculum. Revue des sciences de l'éducation, 28(1), 39-62.

SALOVEY, P. et MAYER, J. D. (1990). Emotional intelligence. Imagination, Cognition, and Personality, 9, 185-211.

SAUVÉ, F. (2012). Analyse de l'attrition des enseignants au Québec (Mémoire de maîtrise inédit). Université de Montréal.

SELYE, H. et TURCOTTE, P. (1976). La gestion du stress. Relations industrielles / Industrial Relations, 31(4), 609-616.

ST-VINCENT, L.-A. (2008). Le pouvoir de l'enseignant messager. Vie pédagogique, (146), 78-83. Récupéré le 5 juillet 2013 de http://www.mels.gouv.qc.ca/sections/ viepedagogique/146/index.asp?page=dossierE_4

VOIROL, C. (1998). Il y a «enseignant» et «ENSEIGNANT». Document inédit, Université de Neuchâtel, Suisse.

WESTEN, D. (2000). Psychologie, pensée, cerveau et culture (2 éd.). Paris: De Boeck Université. 